\title{
Synthesis of a pyrrolo[1,2-a]azepino ring aiming the synthesis of Stemona alkaloids.
}

\section{Luis H. G. Defante*, Ronaldo A. Pilli.}

\begin{abstract}
Aiming the synthesis of the fragment pyrrolo[1,2-a]azepino found in Stemona alkaloids, two methods of synthesis using well-known reactions were examined.
\end{abstract}

\section{Key words:}

Stemona alkaloids, total synthesis, natural products

\section{Introduction}

Herbal extracts containing alkaloids have been used in Asian folk medicine for thousands of years. ${ }^{1}$ One family of these alkaloids is represented by the Stemonaceae alkaloids which are used to suppress coughing and are claimed to present biological activity against some bacteria, fungus and helminths. ${ }^{2}$ Stemoamide is one of the best known representative (Image 1).<smiles>C[C@H]1C(=O)O[C@H]2CCCN3C(=O)CC[C@H]3[C@H]21</smiles>

Image 1. Stemoamide

Thus, this work aims to prepare the fragment pyrrolo[1,2a]azepino present in the alkaloids of the Stemonacae family employing well-known reactions previously described in the synthesis of others natural products. With this adduct in hand, we would be able to proceed with the synthesis of some Stemona alkaloids.

\section{Results and Discussion}

The project is a continuation of an earlier project where we have tried to synthesize the same fragment 8 using a coupling reaction between an acyliminium ion and a nitro compound which did not provide the desired product (Image 1).

$$
\stackrel{\mathrm{R}_{1}}{\mathrm{NO}_{2}}+\underset{\oplus_{\mathrm{R}^{\prime}}^{\prime}}{\stackrel{\text { Lewis acid }}{\longrightarrow}}
$$

2<smiles>[R]C(C1CCCN1[R])[N+](=O)[O-]</smiles>

3
Image 1. Attempt to react a nitro compound with a acyliminium ion.

Facing these results, we decided to change the route (Image 2) and to start a new route from L-pyroglutamic acid (4) that already displays the desired stereogenic center and the pyrrol ring.

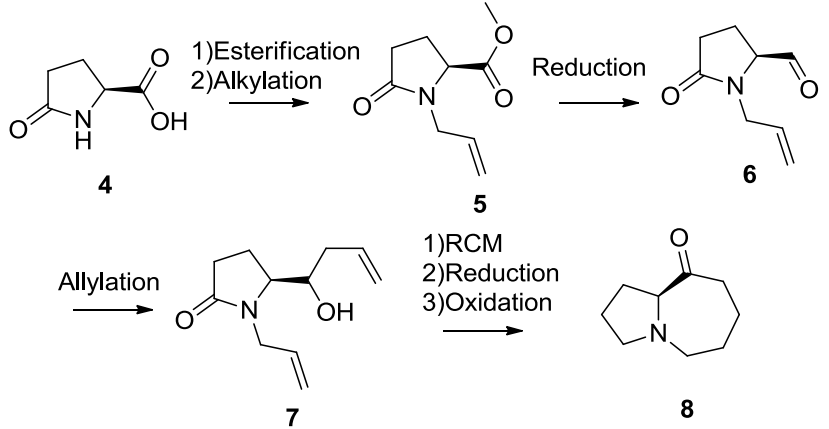

Image 2. Synthetic plan to synthesize fragment $\mathbf{8}$.
Unfortunately, we faced problems in the alkylation step, and the proposed route to fragment 8 could not be implemented.

We are now evaluating a new synthetic route which employs L-proline as the starting material (Image 3).

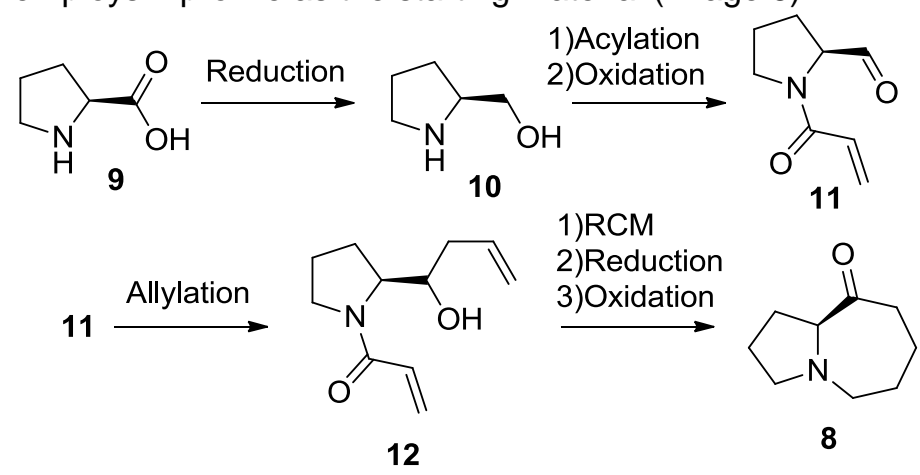

Image 3. Proposal of synthesis

Reduction of L-proline (9) is expected to provide alcohol 10 which is planned to undergo acylation, followed by oxidation, to provide aldehyde 11. Allylation under Grignard conditions should provide homoallylic alcohol 12 which should undergo ring closing metathesis (RCM) to provide the desired bicyclic lactam 8.

\section{Conclusions}

Our first efforts in search of the synthesis of the fragment pyrrolo[1,2-a]azepino 8 using well-known reactions were unsuccessful. We are now studying another route from Lproline and uses similar reactions.

\section{Acknowledgement}

The authors thank the São Paulo Research Foundation (FAPESP), the Brazilian Council for Scientific and Technological Development (CNPq) for financial support and the Institute of Chemistry for facilities support.

${ }^{1}$ H.-S. Chung, P.-M. Hon, G. Lin, P. P.-H. But and H. Dong, Planta Med., 2003, $69,914$.

${ }^{2}$ R. A. Pilli, G. B. Rosso, M. da C. F. de Oliveira, Nat. Prod. Rep.; 2010; 27. 1908. 